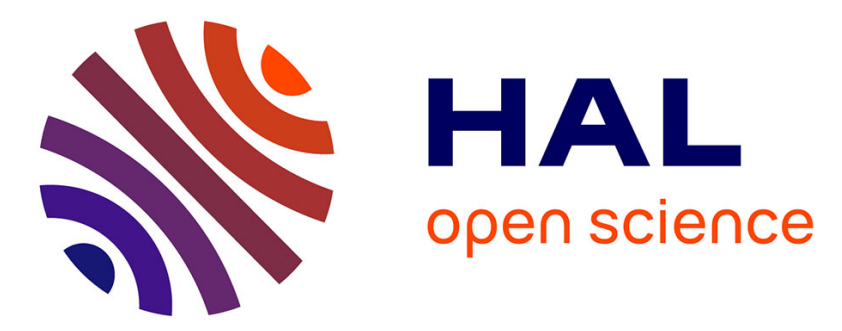

\title{
Force control for tissue tensioning in precise robotic laser surgery
}

S Portolés, P. Vanbiervliet, B. Rosa, C. Tomassetti, C. Meuleman, E.B. B Vander Poorten, D. Reynaerts

\section{- To cite this version:}

S Portolés, P. Vanbiervliet, B. Rosa, C. Tomassetti, C. Meuleman, et al.. Force control for tissue tensioning in precise robotic laser surgery. 2015 IEEE International Conference on Robotics and Automation (ICRA), May 2015, Seattle, France. 10.1109/ICRA.2015.7139237 . hal-01765121

\section{HAL Id: hal-01765121 \\ https://hal.science/hal-01765121}

Submitted on 12 Apr 2018

HAL is a multi-disciplinary open access archive for the deposit and dissemination of scientific research documents, whether they are published or not. The documents may come from teaching and research institutions in France or abroad, or from public or private research centers.
L'archive ouverte pluridisciplinaire HAL, est destinée au dépôt et à la diffusion de documents scientifiques de niveau recherche, publiés ou non, émanant des établissements d'enseignement et de recherche français ou étrangers, des laboratoires publics ou privés. 


\title{
Force Control for Tissue Tensioning in Precise Robotic Laser Surgery
}

\author{
S. Portolés ${ }^{1}$, P. Vanbiervliet, B. Rosa ${ }^{1}$, C. Tomassetti ${ }^{2}$, C. Meuleman ${ }^{2}$, E.B. Vander Poorten ${ }^{1}$, D. Reynaerts ${ }^{1}$
}

\begin{abstract}
Lasers are being used in various surgical procedures to remove tissue or bones, to coagulate vessels or other structures. Due to difficulties in handling only a limited number of surgeons manage to display sufficient levels of precision in Minimally Invasive Surgery (MIS) procedures. Prior works on robotic laser surgery demonstrated shorter learning curves and higher ablation precision, but unfortunately ignored the fact that most clinically relevant tasks are bi-manual by nature. Surgeons are also reluctant to use current commercial surgical robotic systems for complex laser tasks, indicating that the lack of haptic feedback prevents them from efficient and safe tissue handling in preparation of laser treatment. This paper expands earlier robotic laser work towards bi-manual operation. The paper introduces a system for precisely tensioning tissue that is being targeted by the laser. An artificial test-setup that captures some essential features of bimanual laser surgery is described. Experiments have been conducted to investigate the effect of haptic feedback on ablation performance. A comparison is made of achievable levels of ablation precision when there is no haptic feedback, when there is haptic feedback and when an automatic tension control algorithm is deployed. The conducted experimental results confirm the great potential of haptic feedback and automatic tensioning systems for complex bi-manual lasering tasks.
\end{abstract}

\section{INTRODUCTION}

Lasers are being used in various surgical procedures to incise, coagulate or vaporize tissue [1], [2]. Since the ablation or coagulation process takes place without physical contact and the target location can be visualised, e.g. by a low-power pointing laser, before actually ablating, surgeons have great control over the removal process. Compared to cauterisation energy can be delivered in a more focused manner so that collateral damage, damage of surrounding healthy tissue due to overheating, can be limited [3].

In Minimally Invasive Surgery (MIS) lasers are being used progressively [2]. Here, a number of constraints that are typically associated with keyhole surgery make precise laser control inherently more complex. Complicating factors include $i$ ) the loss of direct vision upon the surgical site; $i i$ ) a reduced dexterity as the entry-port constrains instrument motion to 4 DoFs (Degrees of Freedom, in the absence of distal DoFs); iii) instruments need to pivot about their entry-ports which causes motion inversion and additionally creates $i v$ ) a leverage effect. Because of the leverage effect it becomes more difficult to achieve the same motion precision when an instrument is inserted deeper into the body. Physiological tremor will be amplified in such case. Obviously, the longer the procedure, the greater the effect of physiological tremor.

\footnotetext{
${ }^{1}$ KU Leuven, Mechanical Engineering Department, 3001 Leuven, Belgium Sergio.PortolesDiez@kuleuven.be

2 UZ Leuven, Gynaecology and Obstetrics Department, 3000 Leuven, Belgium
}

The chance for making a mistake or inappropriate gesture rises also with time and fatigue [4], [5], [6]. v) Although the lasering process itself does not require contact with the tissue, laser laparoscopy is in fact a bi-manual task. Surgeons use a second forceps to manipulate the targeted tissue. Tissue is being grasped and palpated in order to identify its nature, to align it with respect to the laser beam, but it is also being tensioned to obtain a better control over the exact piece of tissue that will be ablated and over the ablation process itself. Friction forces that develop when sliding instruments in and out the entry-port, mask the interaction (tensioning) forces. The reduced dexterity further limits the options to approach and tension tissue correctly. In short a great deal of skill is needed to prepare the tissue under such conditions of reduced haptic senses and limited dexterity. As a consequence long learning curves have been reported for this type of procedures [7], [8].

Prior work on robotic laser surgery demonstrated shorter learning curves and higher precision for ablation tasks when a robot-mounted laser was controlled e.g. by an intuitive writing interface [9], [10], [11]. However, these works made abstraction from the fact that many of these laser tasks are bi-manual by nature. Procedural efficiency could be severely limited if a second surgeon or assistant is needed aside from the surgeon operating the laser to position and orient the tissue properly. Therefore, this paper expands earlier work on robotic laser surgery towards bi-manual operation.

After introducing a typical and challenging clinical procedure of endometriosis in section II, the envisioned bimanual teleoperation system is sketched in section III. The paper then introduces a system for tension control to control the tissue to receive laser treatment. An artificial experimental setup that was designed to verify the performance of laser operation is described next in section IV. The experimental results are discussed in section V. The paper closes with a conclusion and a view upon further work (section VI).

\section{MIS LASER TREATMENT OF ENDOMETRIOSIS}

Endometriosis is a gynecological condition that affects 6 to $10 \%$ of the general female population [12]. It is characterized by a spreading of cells from the endometrium outside of the uterine cavity. This can cause severe pelvic pain, scarring and adhesions in the abdominal cavity, ultimately leading to infertility. Possible treatments include medication, hormone treatment and surgery. Surgery can be carried out in a laparotomy setup or, in a less invasive way, through a laparoscopic intervention with a $\mathrm{CO}_{2}$ laser or cauterisation tool to ablate endometrial tissue. Latter approaches have been found to be more efficient, and 


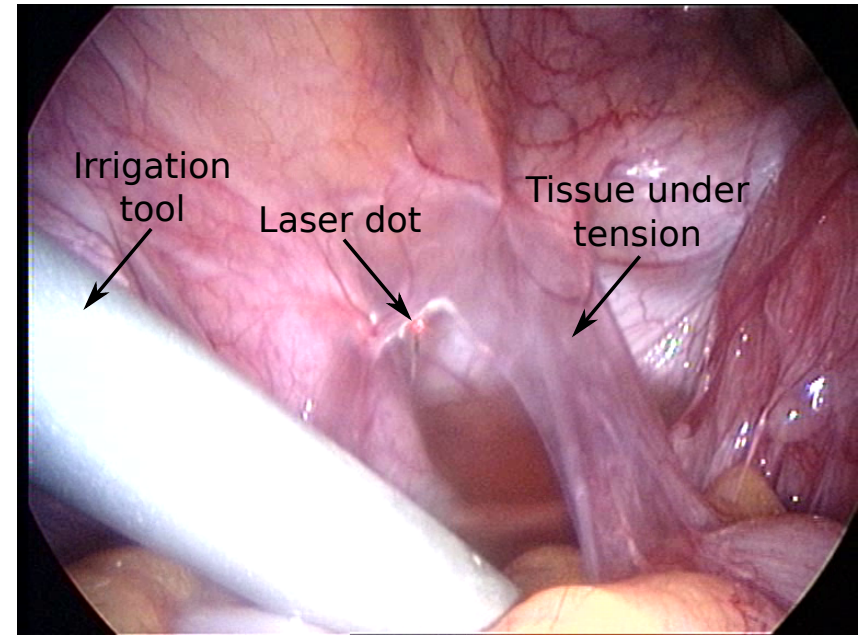

Fig. 1. Cutting of a portion of the peritoneum during endometriosis surgery. The laser dot is visible in the center of the image, and an irrigation tool is being used to tension the tissue, keeping it at a correct angle with respect to the $\mathrm{CO}_{2}$ laser beam.

are now the treatment of choice. Whereas some surgeons advocate laser treatment [13], [14], [15], others prefer cauterisation.

During laser laparoscopy, instruments are inserted through keyholes in the abdominal wall. Classically, a dedicated laparoscope is used, in which the $\mathrm{CO}_{2}$ laser is coaxial with the viewing lenses. This method allows to remove diseased endometrial tissue without touching it, thus avoiding the formation of new adhesions which could be problematic for the patient. However, there are some complicating factors to be accounted for. In order to obtain the best cutting conditions for the laser, it must be kept at a given distance, usually $2 \mathrm{~cm}$, of the endometrial tissue, and oriented normal to it. To maintain the desired distance surgeons must constantly adapt to breathing motion which can become quite tiring over time. Fatigue can lead to increased tremor and imprecision. A second instrument is needed to align the tissue normal to the laser beam. Figure 1 shows an irrigation tool being used to precisely orient and tension the tissue to perform the cut with the $\mathrm{CO}_{2}$ laser. Coordinated (including force) control of all these instruments requires considerable skill. Furthermore interventions are time-consuming and tiring [16].

\section{TISSUE TENSIONING CONTROL}

This section reports on the system that was developed for bi-manual and bi-lateral (i.e. including haptic feedback) robotic laser surgery. After explaining the particularities of the tensioning tool (subsection III-A), the employed bilateral teleoperation control scheme and robotic hardware (subsection III-B), a force measurement system (III-C) and a method for automatic tensioning (subsection III-D) are discussed.

\section{A. Irrigation tool for tissue tensioning}

A wide variety of tools is currently available on the market to grasp and manipulate tissue. At the high-end of

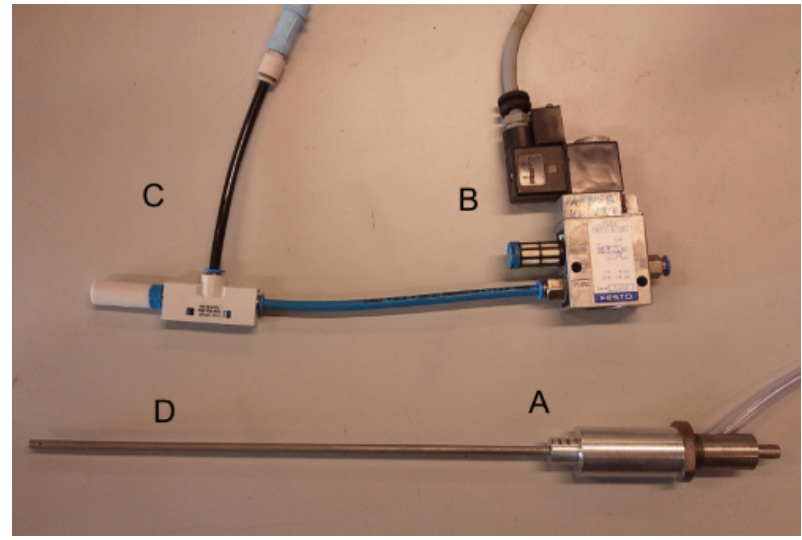

Fig. 2. Different parts of the vacuum gripper: (A) tool holder; (B) electric valve; (C) vacuum pump; (D) Smokevac surgical tool.

the spectrum are sophisticated multiple DoF systems like the JAiMY ${ }^{\mathrm{TM}}$ (Endocontrol) or the EndoWrist ${ }^{\circledR}$ used in Intuitive Surgical's system. For sake of simplicity, rather than equiping and interfacing such fully-fledged forceps, it was opted here to use a simple vacuum-based gripper based on a Venturi effect. Such gripper (Smokevac, Davoll Inc.) is commonly used for irrigation, manipulation and blunt dissection in laparoscopic procedures. By simply controlling the vacuum level, the suction force and similarly the maximal allowable tensioning force can be regulated. A suction cup was mounted at the instrument's tip to increase the diameter (from an original diameter of $3.8 \mathrm{~mm}$ to $8 \mathrm{~mm}$ ) and by doing so raising the achievable suction force (from $0.9 \mathrm{~N}$ to $4 \mathrm{~N}$ at $80 \%$ vacuum). The attainable suction force approaches forces that were found from tensioning experiments conducted on an animal colon. Visser et al. reported here average and maximal tensioning forces of $2.5 \mathrm{~N}$ and $5 \mathrm{~N}$ respectively [17]. Limited research has been conducted so far to study the effect of low pressure on internal organs, but at least Vonck et al. showed that vacuum levels of $60 \%$ do not permanently damage tissue [18]. A gas ejector pump was selected and interfaced to depressurize the instrument and tubing (total volume of 3.4 and $56.7 \mathrm{ml}$ respectively) in less than $200 \mathrm{~ms}$. The motion required during the experimental part is about 30 $\mathrm{mm}$ while the workspace of the platform reaches an insertion distance of $10-26 \mathrm{~cm}$ and allows to reach a circular cone with a vertex angle of $60^{\circ}$. Figure 2 gives an overview of the resulting instrument. The tool holder allows attaching the instrument to the surgical robot (discussed in subsection III-B). By hinging the instrument inside a force measuring trocar (discussed in III-C) it becomes possible to estimate the actual interaction (including suction) force with the tissue.

\section{B. Basic teleoperation platform and control}

For the development of a tensioning system the in-house developed LoTESS platform, displayed in Fig.3, was upgraded. The LoTESS platform is a multi-purpose 3-DoF robot that was designed to study bilateral control schemes for MIS applications[19]. The platform consists of two dynamically similar mechanisms that provide a 3 -dimensional 


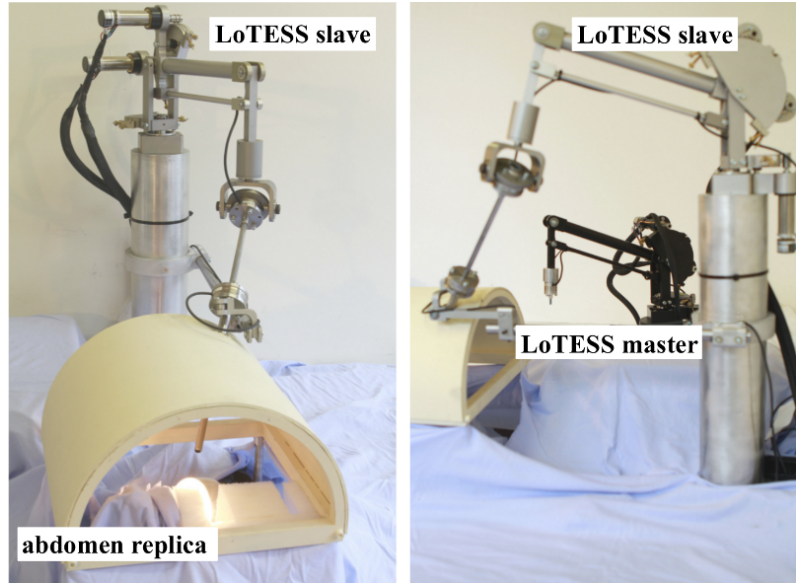

Fig. 3. View upon LoTESS teleoperation system used to manipulate the irrigation tool providing haptic feedback to its user.[19].

cylindrical workspace. The irrigation tool is controlled by the slave robot. It is hinged by a pair of passive joints at the slave's end-effector at one side and a passive stabilising mechanism, that is to be aligned with the entry-port in the patient's body, at the other side. The system is programmed so as to map the pose of the master joystick's handle to the tip of the irrigation tool (inside the patient) thus effectively cancelling out the problem of motion inversion.

The different elements of the system are linked through OROCOS, an in-house developed component-based rea-ltime robotic middleware [20] running on a pre-empted Linux kernel. The different current-controlled actuators (brushless DC motors), encoders, foot pedals and the proportional valve for suction control are interfaced by means of a Beckhoffbased data acquisition system and a National Instrument PCI card in the main computer. An EtherCAT communication protocol is set up between the main computer and the Beckhoff system allowing fast and real-time data transmission. All acquisition, computations (e.g. forward and backwards kinematics) control and communication happens at a rate of $5 \mathrm{kHz}$.

Different combinations of controllers have been implemented including a simple uni-lateral feedforward controller (whereby the master position is sent to a position-controller at the slave side), and a 3-channel bi-lateral controller [21], [22], discussed in more detail in subsection III-C. Additionally some gravity compensation schemes are running at the master and slave side so that no expected motion occurs in case the user would release the system.

\section{Force measurement and haptic feedback}

Force control (discussed in subsection III-D) and haptic feedback can only be implemented properly if an adequate force sensing capability is present. The interaction between the operator and the master robot is here measured by a 6-axis force/torque sensor (ATI Industrial Automation) attached to the end-effector of the manipulator. For the slave manipulator the situation is a bit more complicated as sterility issues are to be considered when considering tip-mounted

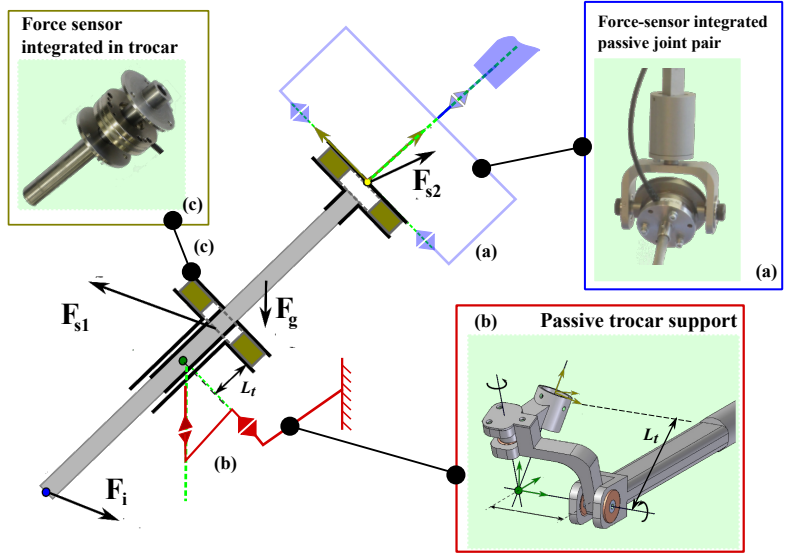

Fig. 4. Extra-corporeal force measurement system featuring (a) force sensor-integrated passive joint at the end-effector; (b) a passive trocar support with (c) trocar with integrated force sensor.

force sensors. On the other hand, if a force sensor would be simply mounted between the robot's end-effector and the instrument, it would be difficult to distinguish interaction forces from friction forces that develop at the trocar. For this reason an extra-corporeal force measurement system presented earlier by Willaert et al. [23] is adopted here. This solution facilitates further in-vivo testing.

Fig. 4 gives a schematic view upon the force measurement system. The instrument is hinged between two extracorporeal 6-axis force transducers. A first force sensor is inserted between the instrument and the robot's end-effector permitting free instrument rotation in 2 DoFs. A second sensor is inserted between the instrument and a passive trocar support. At both sides, a pair of passive joints ensure absence of bending torques (apart from the torque about the instrument's axis which is assumed small). Not only do these passive joints help prevent sensor overload, they also make the instrument pivot about a remote center of motion. If properly aligned with the entry-port into the body this ensures minimal tissue stress at the body wall. In an equilibrium state the interaction force $F_{i}$ can be computed straightforwardly from a free-body analysis applied on the suction tube, including force measurements $F_{s 1}$ and $F_{s 2}$. Hereby, it is important to account for the effect of gravitional forces $F_{g}$, which can be derived from knowledge of the instrument's pose and respective pose of the center of gravity.

The estimated interaction forces are then used in a PD$\mathrm{F}_{-} \mathrm{F}_{e}$ 3-channel teleoperation controller, discussed in greater depth in [24]. This PD-F-F $F_{e}$ controller sends the master joystick's position $x_{h}$ and forces $F_{h}$ applied by the human operator as set-points for local controllers at the slave side. The interaction force $F_{e}$ between slave and environment is reflected back to the master. The PD-F-F $F_{e}$ presents an acceptable trade-off between overall transparency and stability. To improve robustness of the controller, the position is scaled down by a factor $\mu_{h}=0.6$. By augmenting the force from the slave by $\lambda_{e}=1.5$ it is made easier for the user to discriminate between force levels. Note that increasing the force reflection 


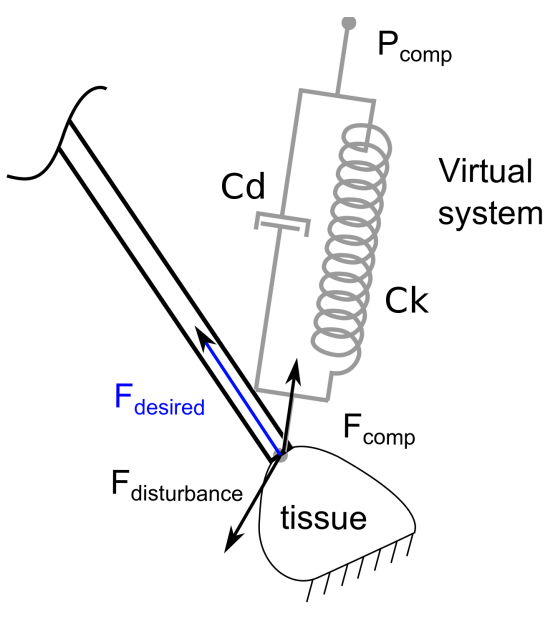

Fig. 5. Schematic of the developed autotensioning controller. A proxy point $P_{\text {comp }}$ serves as a reference for an impedance controller (impedance given by $C_{k}$ and $C_{d}$ ). By displacing $P_{\text {comp }}$ it is possible to compensate for moderate disturbance forces and maintain a target tension level.

further would lead to instability.

\section{Automatic tensioning}

A third type of control, automatic tensioning, was implemented additionally. In this scenario, the user is asked to indicate a desired force level after which the system will try to automatically maintain the specified tensioning force upon the tissue. The automatic tensioning is only a part of a procedure. A Finite State Machine (FSM) was made to cycle through the different system states. During approach and tensioning the abovementioned PD-F-F $F_{e}$ controller is used. Once the tissue tension is appropriate, the user indicates this by pressing a foot pedal upon which the system switches to the constant tension control state. Fig. 5 displays the impedance controller (impedance parameters $K_{c}$ and $K_{v}$ ) that was developed to maintain the target tension in the face of possible disturbance forces.

\section{EXPERIMENTAL SETUP AND PROTOCOL}

Some complex laser surgical tasks, for instance for endometriosis surgery, are generally not recommended by surgeons for robotic-assisted interventions [25]. Lack of haptic feedback and control over the tensioning force is a main reason for the choice of the more classical laparoscopy [26]. It is important to investigate whether there is indeed a fundamental performance issue and whether haptics or force control do actually fulfill their expectations.

To answer these questions and quantify the influence of the proposed haptic and force control schemes a simple somewhat artificial, but arguably representative setup was designed. The setup was inspired by similar artificial setups that are used for accrediation such as FLS certification or MISTELS [27], but was tailored to measure the performance of bi-manual laser surgical tasks.

\section{A. Description of the experimental setup}

Fig.6 shows the overall layout of the setup that was designed. The setup consists of following elements: (1) an

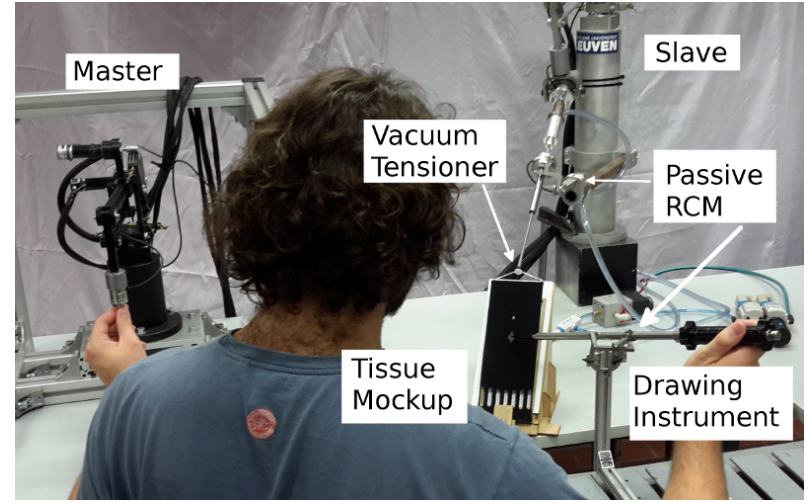

Fig. 6. Top view of the layout of the setup displaying the user, the teleoperation system, artificial tissue, laparoscopic drawing instrument hinged in their respective passive remote centers of motion.

artifical mockup consisting of elastic material that replicates tissue that is to be tensioned and processed (for this experiment a $23 \mathrm{~cm} \times 11 \mathrm{~cm}$ cloth of a cotton and elastane was used); (2) a teleoperation system to tension the 'tissue'. The master is located at the left hand of the subject who uses it to tension the tissue. The slave with vacuum-based tensioning tool is placed directly in front of the subject; (3) a laparoscopic instrument with a liquid ink marker attached at its end mimicking the behavior of a laser laparoscope (right hand of the subject); (4) a passive 2-DoF hinge that simulates the entry point where the trocar is entering the thoracic or abdominal cavity. The subject is asked to pass the 'laser laparoscope' through this hinged trocar and as such is forced to cope with traditional challenges such as limited access, motion inversion or changing of leverage.

\section{B. Surgical task - laparoscopic laser scanning}

An experiment was designed to approximate typical laparoscopic laser scanning tasks where surgeons need to scan and laser an enclosed surface area of tissue while keeping it under tension. For simplicity, subjects are asked here to colour a predefined shape on a paper that is positioned right below the artificial tissue (instead of lasering it). A diamondlike pattern was laser-cut out of the fabric so that when under a certain targeted tension the deformed diamond shape is well-determined and repeatable. To validate the capability of the subject to simultaneously laser and tension tissue we investigate the shape of the surface that is marked by the subject. In case of a perfect execution the coloured region should match exactly the shape of the deformed diamond (under the targeted tension). To help the user setting the targeted tension a small circular hole and reference dot on the underlying paper was made. By visually aligning both dots the user knows that the targeted tension is reached. Visual information is also used in typical laser surgery, but the hypothesis made here is that with visual information only the lasering quality and/or efficiency is inferior. To simplify performance evaluatioon a number of fiducial markers are printed on top of the paper sheet that allow easy alignment and computation of the results through image processing. 


\section{Experimental Protocol}

User experiments were carried out to compare the performance of the bi-manual 'laser' task where for tissuetensioning one of three different feedback modalities are offered: $a$ ) visual feedback only (teleoperation without haptic feedback), b) bi-lateral teleoperation feedback and $c$ ) with force feedback and autotensioning feature.

Each time the following steps had to be performed:

1) hold the master robot;

2) teleoperate the tensioning tool and grasp the tissue;

3) apply the correct amount of tension (by aligning the hole with the circular mark on the underlying paper sheet);

4) press the foot pedal to indicate your intention to start the drawing task (and keep it pressed while drawing);

5) mark the entire region of the deformed diamond-like cut-out on the underlying paper sheet;

6) indicate to the examiner when your task is ended and release the pedal.

In cases were grip on the tissue was lost e.g. due to application of excessive tensioning forces or simply by having a bad grip on the tissue, the user was allowed to restore the tissue by hand and to grip the tissue again (step 2) with the teleoperation and continue the task execution. During such recovery periods users had to release their foot from the pedal to mark these events for later analysis.

Subjects were explained the procedure vocally, but the examiner also demonstrated the procedure once per subject. After the demonstration the subjects could try out each of the three different operating modalities to get used to them. This also helped them memorize the different steps of the protocol. During preliminary tests a certain learning effect was found from simple visual analysis of the patterns. Therefore, the first trials of each user were excluded from further analysis. Users had to conduct 12 tests ordered in 3 batches of a certain randomly presented modalities.

\section{Performance Analysis}

The fully sensorized robotic platform provides access to a large amount of data that can be used to analyse the overall performance: position and forces exerted by the user, movement of the tensioning tool and tension applied on the tissue. The status of the foot-pedal tells whether the user is painting, is still in preparation or lost his/her grip on the tissue. Also, the performance of the 'lasering' task can be inspected visually from the marks made on the paper sheets. The fiducial points on the sheets were matched to those of the reference using SiFT features in order to align them together using a homography. Then, the area of interest was compared. In order to assess the performance of the user while drawing, 3 metrics were computed, the precision $P$, the recall $R$ and the centroid shift $C_{s}$. The latter is simply the position shift between the centroid of the master pattern and the centroid of the drawn pattern. It is, indirectly, an image of the tensioning quality of the experiment. If $\mathscr{D}$ is the set of pixels drawn by the user, and $\mathscr{M}$ the set of pixels of the master pattern, then $P$ and $R$ can then be defined as:

$$
\begin{aligned}
P & =\frac{\mathscr{D} \cap \mathscr{M}}{\mathscr{D}} \\
R & =\frac{\mathscr{D} \cap \mathscr{M}}{\mathscr{M}}
\end{aligned}
$$

The precision can be seen as a measure of the quality of the drawing and the recall as a measure of the completeness of the drawing.

\section{EXPERIMENTAL RESULTS}

10 users participated in the experiments. $80 \%$ percent of the participants were male, $20 \%$ were female, $80 \%$ were right-handed, $20 \%$ left-handed. Participant age ranged from 23 to 29 years. 3 of the users had prior experience with haptic systems 7 had no experience whatsoever. None of the users had surgical expertise. A number of different performance metrics were calculated from the gathered data. The statistical values of the different computed metrics, grouped per type controller, are summarized in Table I. The normallity of the distributions of the different metrics was checked with Lilliefors test rejecting the hypothesis for many of the cases. For the non-parametric distributions, the equality between medians was checked by applying the Kruskal-Wallis test. The samples for which normal distribution can be assumed were compared by means of ANOVA tests.

The 'overall time' is counted from the signal given by the user that he/she is ready to start the experiment until the user indicates to be ready when the drawing task has been completed. The assembled data shows evidence of an increase in overall time for basic teleoperation with respect to execution with haptic feedback or force control $(p<0.05)$.

The 'drawing time' refers only to that portion of time that the user is actually drawing. This portion is communicating by keeping the foot-pedal pressed. For the drawing time no significant difference can be observed between the different groups which suggests that the users were rather slow during the tensioning time. On the other hand instrument movements were more precise when putting the tissue under tension in preparation of the drawing task for haptic modalities compared to the non-haptic case as the 'manipulation time' indicates.

Another metric that highlights this phenomenom is the 'number of tissue drops' (grip on tissue is lost and regrasp is needed). While a single drop was the maximum for the cases with haptics and force control, the median for experiments without force feedback was two. An example of the different cases and the number of drops is depicted in Fig. 7. At second 12 the tissue is dropped exactly at the moment that the user decided to start painting. Note, that the area marked in green in the figures corresponds to the time where the foot pedal is pressed and the users draws upon the underlying sheet. The delay between green area and tissue loss corresponds to the reaction time of the user to release the foot-pedal after noticing loss of grip.

The mean 'interaction forces' do not show significant differences among groups, however a very strong evidence 

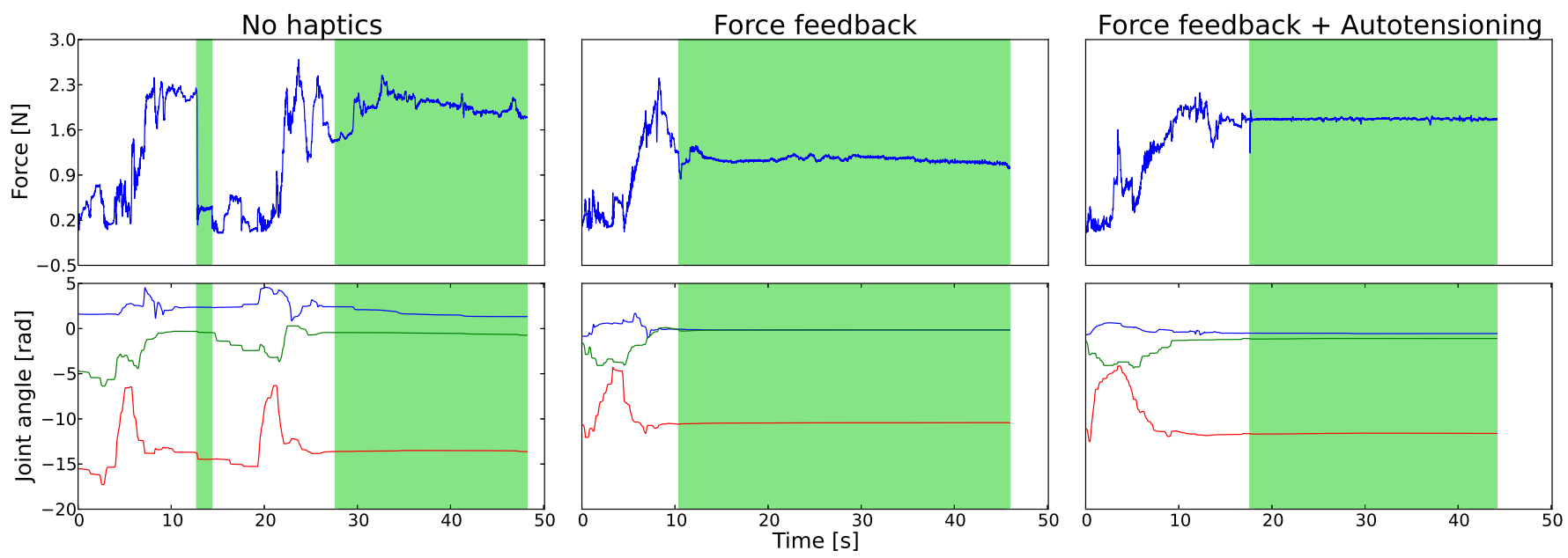

Fig. 7. Time series for one arbitrary experiments of each type. Upper graphs display the Norm of the force at the tip of the tensioning tool. Lower graphs depict the evolution of all the three joint angles of the slave robot. Green areas denote the intention of the user to perform the right-hand task revealed by means of pressing the foot-pedal.

was observed regarding the difference in 'standard deviation on forces'. This effect can be easily observed from the recordings in Fig. 7. The upper diagram shows here the evolution of the tensioning force during drawing task (parts with green background). As expected an extremely steady behaviour can be seen for the autotensioning controller. This behaviour can also be seen on the lower plots, that show the displacement of the slave joint(s). On the other hand, even if mean and median values differ between basic teleoperation and force feedback control schemes for standard deviation of the force, there is not enough evidence $(p=0.15)$ to ensure that such deviation is not caused by the random sampling of the population. Forces applied to the tissue vary largely when haptics is missing risking to exceed limits and causing tissue damage.

Large similarities can be observed for the values of "precision' and 'recall'. An interesting observation is that recall values are higher than precision values. This means that users tend to fill the shape completely rather than respecting the boundaries of the targeted domain. Note that this can also be partially explained by the large compliance of the covering tissue. Deviation of the centroids of the drawings in any axis are not significant and event the differences are in some circumstances under the pixel level.

\section{CONCLUSIONS AND FURTHER WORK}

This paper presented a telerobotic setup for improving the precision of laser surgical procedures. The paper starts from the observation that laser surgery is bimanual by nature and that current surgeons are reluctant for employing robotic technology as current systems do not provide haptic feedback. An experimental setup was built to verify this argument. The setup consist of a 3-DoF telesurgical system, an extracorporeal force measurement system. A vacuumbased suction instrument is used to tension tissue. Three different control laws were implemented, a uni-lateral, a bilateral three channel controller and a bi-lateral controller with
TABLE I

STATISTICAL ANALYSIS OF CALCULATED METRIC FOR EACH TEST.

\begin{tabular}{|c|c|c|c|c|}
\hline Metric & & Teleop & F-feedback & F-f + Auto \\
\hline \multirow{4}{*}{$\begin{array}{l}\text { Overall time } \\
p=0.04\end{array}$} & mean & $80.7 \mathrm{~s}$ & $50.9 \mathrm{~s}$ & $56.3 \mathrm{~s}$ \\
\hline & median & $61.5 \mathrm{~s}$ & $51.1 \mathrm{~s}$ & $51.1 \mathrm{~s}$ \\
\hline & $\min$ & $33 \mathrm{~s}$ & $31.4 \mathrm{~s}$ & $35.4 \mathrm{~s}$ \\
\hline & $\max$ & $184 \mathrm{~s}$ & $79.4 \mathrm{~s}$ & $87.5 \mathrm{~s}$ \\
\hline \multirow{4}{*}{$\begin{array}{l}\text { Drawing time } \\
p=0.36\end{array}$} & mean & $31.2 \mathrm{~s}$ & $29.8 \mathrm{~s}$ & $33.8 \mathrm{~s}$ \\
\hline & median & $27 \mathrm{~s}$ & $28 \mathrm{~s}$ & $31.7 \mathrm{~s}$ \\
\hline & $\min$ & $21 \mathrm{~s}$ & $18.4 \mathrm{~s}$ & $22.5 \mathrm{~s}$ \\
\hline & $\max$ & $51.4 \mathrm{~s}$ & $55.6 \mathrm{~s}$ & $50.9 \mathrm{~s}$ \\
\hline \multirow{4}{*}{$\begin{array}{l}\text { Force mean } \\
p=0.82\end{array}$} & mean & $1.39 \mathrm{~N}$ & $1.33 \mathrm{~N}$ & $1.33 \mathrm{~N}$ \\
\hline & median & $1.3 \mathrm{~N}$ & $1.37 \mathrm{~N}$ & $1.41 \mathrm{~N}$ \\
\hline & $\min$ & $0.7 \mathrm{~N}$ & $0.78 \mathrm{~N}$ & $0.74 \mathrm{~N}$ \\
\hline & $\max$ & $2.32 \mathrm{~N}$ & $1.86 \mathrm{~N}$ & $1.93 \mathrm{~N}$ \\
\hline \multirow{4}{*}{$\begin{array}{l}\text { Force std } \\
p<1 e^{-7}\end{array}$} & mean & $250 \mathrm{mN}$ & $180 \mathrm{mN}$ & $30 \mathrm{mN}$ \\
\hline & median & $220 \mathrm{mN}$ & $160 \mathrm{mN}$ & $20 \mathrm{mN}$ \\
\hline & $\min$ & $50 \mathrm{mN}$ & $10 \mathrm{mN}$ & $7 \mathrm{mN}$ \\
\hline & $\max$ & $580 \mathrm{mN}$ & $500 \mathrm{mN}$ & $290 \mathrm{mN}$ \\
\hline \multirow{4}{*}{$\begin{array}{l}\# \text { drops } \\
p<1 e^{-4}\end{array}$} & mean & 1.9 & 0.14 & 0.02 \\
\hline & median & 2 & 0 & 0 \\
\hline & $\min$ & 0 & 0 & 0 \\
\hline & $\max$ & 6 & 1 & 1 \\
\hline \multirow{4}{*}{$\begin{array}{l}\text { Precision } \\
p=0.68\end{array}$} & mean & $79 \%$ & $79 \%$ & $76 \%$ \\
\hline & median & $80 \%$ & $77 \%$ & $75 \%$ \\
\hline & $\min$ & $60 \%$ & $60 \%$ & $61 \%$ \\
\hline & $\max$ & $94 \%$ & $92 \%$ & $91 \%$ \\
\hline \multirow{4}{*}{$\begin{array}{l}\text { Recall } \\
p=0.57\end{array}$} & mean & $93 \%$ & $95 \%$ & $93 \%$ \\
\hline & median & $94 \%$ & $96 \%$ & $96 \%$ \\
\hline & $\min$ & $80 \%$ & $79 \%$ & $83 \%$ \\
\hline & $\max$ & $100 \%$ & $100 \%$ & $100 \%$ \\
\hline \multirow{4}{*}{$\begin{array}{l}\text { Deviation }_{x} \\
p=0.42\end{array}$} & mean & 0.1 pixels & -0.1 pixels & -0.1 pixels \\
\hline & median & -1 pixel & -2 pixels & -2 pixels \\
\hline & $\min$ & -5 pixels & -11 pixels & -6 pixels \\
\hline & $\max$ & 26 pixels & 3 pixels & 3 pixels \\
\hline \multirow{4}{*}{$\begin{array}{l}\text { Deviation }_{y} \\
p=0.99\end{array}$} & mean & -1.9 pixels & -2.6 pixels & -2.23 pixels \\
\hline & median & -3 pixels & -2 pixels & -2 pixels \\
\hline & $\min$ & -6 pixels & -14 pixels & -6 pixels \\
\hline & $\max$ & 8 pixels & 2 pixels & 3 pixels \\
\hline
\end{tabular}


automatic tensioning capability. The level of forces perceived and displacements of the surgical tool have been adjusted on the controller to work in an appropriate workspace in a precise way. An artificial experimental setup and task was designed to replicate a bilateral laser surgical task.

Experiments have been carried out with 10 users to compare the different operating modalities and quantify the effect of haptics or force control in such bi-manual tasks. The results show that haptics is indeed an essential feature for the tissue tensioning task, with statistically significant improvement in both time to completion, number of times tissue grip is lost and larger standard deviations on executed forces. Latter observation suggests that this operation mode is more prone to sudden peak forces/stresses which would increase the risk for tissue damage. The results for haptic feedback are comparable to those for automatic tensioning, however for the latter could free one hand, which would be beneficial especially during complex tasks.

Further work will be conducted to further explore the benefits of haptics/automatic tensioning. This will be done by building up a more realistic robotic system consisting of a pair of master and a pair of robotic slaves, and designing more challenging and complex surgical tasks.

\section{REFERENCES}

[1] A. Luciano, G. Frishman, and D. Maier, "A comparative analysis of adhesion reduction, tissue effects, and incising characteristics of electrosurgery, c02 laser, and nd: Yag laser at operative laparoscopy: an animal study," Journal of laparoendoscopic surgery, vol. 2, no. 6, pp. 287-292, 1992.

[2] M. Adelman, L. Tsai, E. Tangchitnob, and B. Kahn, "Laser technology and applications in gynaecology," Journal of Obstetrics \& Gynaecology, vol. 33, no. 3, pp. 225-231, 2013.

[3] J. Liboon, W. Funkhouser, and D. J. Terris, "A comparison of mucosal incisions made by scalpel, co2 laser, electrocautery, and constantvoltage electrocautery," Otolaryngology-Head and Neck Surgery, vol. 116, no. 3, pp. 379-385, 1997.

[4] L. T. Kohn, J. M. Corrigan, and M. S. Donaldson, To Err Is Human: Building a Safer Health System, I. o. M. Committee on Quality of Health Care in America, Ed. The National Academies Press, 2000.

[5] P. Joice, G. Hanna, and A. Cuschieri, "Errors enacted during endoscopic surgerya human reliability analysis," Applied ergonomics, vol. 29 , no. 6, pp. 409-414, 1998.

[6] B. Tang, G. Hanna, and A. Cuschieri, "Analysis of errors enacted by surgical trainees during skills training courses," Surgery, vol. 138, no. 1, pp. 14-20, 2005.

[7] Z. Holub, A. Jabor, P. Bartoš, J. Hendl, and Š. Urbánek, "Laparoscopic surgery in women with endometrial cancer: the learning curve," European Journal of Obstetrics \& Gynecology and Reproductive Biology, vol. 107, no. 2, pp. 195-200, 2003.

[8] C. Larsen, T. Grantcharov, R. Aggarwal, A. Tully, J. L. Sørensen, T. Dalsgaard, and B. Ottesen, "Objective assessment of gynecologic laparoscopic skills using the lapsimgyn virtual reality simulator," Surgical Endoscopy And Other Interventional Techniques, vol. 20, no. 9, pp. 1460-1466, 2006.

[9] H. Tang, H. Van Brussel, J. Vander Sloten, D. Reynaerts, G. De Win, B. Van Cleynenbreugel, and P. Koninckx, "Evaluation of an intuitive writing interface in robot-aided laser laparoscopic surgery," Computer Aided Surgery, vol. 11, no. 1, pp. 21-30, 2006.
[10] L. S. Mattos, G. Dagnino, G. Becattini, M. Dellepiane, and D. G. Caldwell, "A virtual scalpel system for computer-assisted laser microsurgery," in Intelligent Robots and Systems (IROS), 2011 IEEE/RSJ International Conference on. IEEE, 2011, pp. 1359-1365.

[11] J. Ortiz, L. S. Mattos, and D. G. Caldwell, "Smart devices in robotassisted laser microsurgery: Towards ubiquitous tele-cooperation," in Robotics and Biomimetics (ROBIO), 2012 IEEE International Conference on. IEEE, 2012, pp. 1721-1726.

[12] C. Bulletti, M. E. Coccia, S. Battistoni, and A. Borini, "Endometriosis and infertility," Journal of assisted reproduction and genetics, vol. 27, no. 8, pp. 441-447, 2010.

[13] G. Adamson, L. Subak, D. Pasta, S. Hurd, O. von Franque, and R. Rodriguez, "Comparison of co2 laser laparoscopy with laparotomy for treatment of endometriomata," Fertility and Sterility, vol. 57, no. 5, pp. 965-973, 1992.

[14] C. Sutton and K. Jones, "Laser laparoscopy for endometriosis and endometriotic cysts," Surgical Endoscopy And Other Interventional Techniques, vol. 16, no. 11, pp. 1513-1517, 2002.

[15] P. R. Koninckx and E. McVeigh, "Laser surgery for endometriosis," Modern Management of Endometriosis, pp. 245:1-19, 2005.

[16] R. Garry, "Laparoscopic excision of endometriosis: the treatment of choice?" BJOG: An International Journal of Obstetrics \& Gynaecology, vol. 104, no. 5, pp. 513-515, 1997.

[17] H. Visser, J. Heijnsijk, J. Herder, and P. Pistecky, "Forces and displacements in colon surgery," in Surgical Endoscopy And Other Interventional Techniques, vol. 16, no. 10, October 2002, pp. 14261430.

[18] D. Vonck, "The feasibility of vacuum technique in minimal invasive surgery," Ph.D. dissertation, TU Delft, 2013.

[19] B. Willaert, B. Corteville, J. V. Vlem, K. Vanwynsberghe, D. Reynaerts, and H. V. Brussel, "A multi-purpose haptic device for research on physical human-robot interaction," in 13th International Conference on New Actuators, Bremen, Germany, June 2012, pp. 530-533.

[20] H. Bruyninckx, "Open robot control software: the orocos project," in Robotics and Automation, 2001. Proceedings 2001 ICRA. IEEE International Conference on, vol. 3, 2001, pp. 2523-2528 vol.3.

[21] D. Lawrence, "Stability and transparency in bilateral teleoperation," Robotics and Automation, IEEE Transactions on, vol. 9, no. 5, pp. 624-637, Oct 1993.

[22] K. Hashtrudi-Zaad and S. Salcudean, "Analysis and evaluation of stability and performance robustness for teleoperation control architectures," in Robotics and Automation, 2000. Proceedings. ICRA 'O0. IEEE International Conference on, vol. 4, 2000, pp. 3107-3113 vol.4.

[23] B. Willaert, N. Famaey, P. Verbrugghe, D. Reynaerts, and H. V. Brussel, "Design and in vivo validation of a force-measuring manipulator for mis providing synchronized video, motion and force data," in Robotics and Automation (ICRA), 2013 IEEE International Conference on, May 2013, pp. 4857-4862.

[24] B. Willaert, B. Corteville, D. Reynaerts, H. Van Brussel, and E. Poorten, "Transparency trade-offs for a 3-channel controller revealed by the bounded environment passivity method," in Advances in Computer-Human Interactions, 2010. ACHI '10. Third International Conference on, Feb 2010, pp. 66-72.

[25] M. Catenacci, R. Flyckt, and T. Falcone, "Robotics in reproductive surgery: Strengths and limitations," Placenta, vol. 32, no. 3, pp. S232S237, 2011.

[26] A. Szold, R. Bergamaschi, I. Broeders, J. Dankelman, A. Forgione, T. Lang $\varnothing$, A. Melzer, Y. Mintz, S. Morales-Conde, M. Rhodes, et al., "European association of endoscopic surgeons (eaes) consensus statement on the use of robotics in general surgery," Surgical endoscopy, vol. 29, no. 2, pp. 253-288, 2015.

[27] A. M. Derossis, G. M. Fried, M. Abrahamowicz, H. H. Sigman, J. S. Barkun, and J. L. Meakins, "Development of a model for training and evaluation of laparoscopic skills," The American Journal of Surgery, vol. 175 , no. 6 , pp. $482-487,1998$. 